\title{
Addition of silver nanoparticles reduces the wettability of methacrylate and silorane-based composites
}

\author{
Shahin Kasraei(a) \\ Mohadese Azarsina(b) \\ (a) Department of Operative Dentistry, Dental \\ Research Center, Dental School, Hamadan \\ Univ of Medical Sciences, Hamadan, Iran. \\ (b) Department of Operative Dentistry, Dental \\ School, International Branch of Shahid \\ Beheshti Univ of Medical Sciences, \\ Tehran, Iran.
}

\begin{abstract}
Incorporation of silver nanoparticles into composite resins is recommended for their reported antibacterial properties, but this incorporation can affect the wettability of such materials. Therefore, this study evaluated the effect of nano-silver addition to silorane-based and methacrylate-based composites on their contact angle. Nano-silver particles were added to Z250 (methacrylate-based) and P90 (silorane-based) composites at $0.5 \%$ and $1 \%$ by weight. The control group had no additions. SEM-EDX analysis was performed to confirm the homogeneity of the nano-silver distribution. Seventy-two composite discs were prepared and standardized to the identical surface roughness values, and then distributed randomly into 6 groups containing 12 samples each $(\mathrm{N}=12)$. Two random samples from each group were observed by atomic force microscopy. Distilled water contact angle measurements were performed for the wettability measurement. Two-way ANOVA, followed by the TukeyHSD test, with a significance level of $5 \%$, were used for data analysis. It was observed that wettability was significantly different between the composites $(\mathrm{p}=0.0001)$, and that the addition of nano-silver caused a significant reduction in the contact angle $(\mathrm{p}=0.0001)$. Wettability varied depending on the concentration of the nano silver $(\mathrm{p}=0.008)$. Siloranebased composites have a higher contact angle than methacrylate-based composites. Within the limitations of this study, it can be concluded that the addition of $0.5 \%$ nano-silver particles to the composites caused a decrease in the contact angle of water.
\end{abstract}

Descriptors: Nanotechnology; Silver; Composite Resins; Hydrophobic and Hydrophilic Interactions.

\section{Introduction}

Silorane composites are a new generation of restoratives with a ringopening cationic polymerization mechanism..$^{1-3}$ These restorative materials have been introduced in an attempt to overcome the polymerization shrinkage of conventional methacrylate-based composites. A low potential to absorb the dyes from daily nutrition is considered to be an advantage of silorane-based restoratives because of their siloxane backbone, which is responsible for the hydrophobic nature of the restorative material. ${ }^{3,4}$

Measurement of the contact angle at the solid-air-liquid meeting point is a widely known technique used to investigate wettability of solid substrates. Lower wettability of the composite resin surface and less wa-
Submitted: May 16, 2012

Accepted for publication: Aug 18, 2012

Last revision: Sep 20, 2012 
ter sorption contribute to the reduction of staining, and is an effective factor affecting plaque accumulation. ${ }^{5,6}$ Silorane-based composites are claimed to have a higher contact angle and a lower amount of plaque adhesion compared to methacrylate-based composites. ${ }^{7}$

In operative dentistry, secondary caries is the main reason for restoration replacement. ${ }^{8}$ Therefore, dental materials with low wettability properties are preferable to limit the adhesion and proliferation of pathogens and, consequently, to prevent secondary caries. Although the presence of silver in restorative materials has proved to be effective against streptococci of the human oral cavity, and it might also be useful as an antibacterial additive to dental restorations, ${ }^{9}$ it is not known whether the addition of silver at the nano scale could interfere with the wettability of dental restorative materials.

Furthermore, considering that the incorporation of nanoparticles can affect restoration contact angle measurements, ${ }^{10,11}$ the aim of the present study was to evaluate the effect of the addition of nano silver particles to silorane-based and methacrylate-based composites on this surface property.

\section{Methodology \\ Experimental design}

This experimental study was performed on 72 resin composite discs. Nano silver particles (TopNano Tech Co., Taipei, Taiwan) with an average size of $50 \mathrm{~nm}$ were added mechanically to Z250 (3M ESPE, St. Paul, USA) and P90 (3M ESPE) composites at $0.5 \%$ and $1 \%$ by weight. The control group did not contain any silver. One sample from each test group was prepared for SEM-EDX analysis. Each sample was observed with a scanning electron microscope (Tescan, VegaII, XMU, Brno, Czech Republic) at $350 \times$. To assess the wettability of the samples distilled water contact angles on composite resins were measured using a contact angle measuring system. Two-way ANOVA, followed by Tukey-HSD, were used for data analysis. The significance level was set at $5 \%$.

\section{Specimen preparation}

Nano silver particles (TopNano Tech Co., Tai- pei, Taiwan) with an average size of $50 \mathrm{~nm}$ were added mechanically to Z250 (3M ESPE) and P90 (3M ESPE) composites at $0.5 \%$ and $1 \%$ by weight. Unloaded composite resins were used as controls. The nano particles were mixed continuously with the composites using a plastic spatula for $30 \mathrm{~min}$ in a dark room. The samples were prepared in PVC molds of $10 \mathrm{~mm}$ diameter and $1.5 \mathrm{~mm}$ height. The composite was inserted into the mold and immediately covered with two glass slides from the top and the bottom. The specimen was polymerized by light, using an LED (Demi LED Light Curing System, Kerr Corp., Orange, USA) light-curing unit with a light intensity of $800 \mathrm{~mW} / \mathrm{cm}^{2}$, measured with a radiometer for $60 \mathrm{~s}$ from both sides. All of the samples were polished with 600,800 , and 1200 grit SiC papers (991A Softlex, Berlin, Germany) to obtain highly polished samples with identical surface roughness $(\mathrm{Ra})$ values. To confirm the $\mathrm{Ra}$ was homogenous, two samples from each group were observed randomly by atomic force microscopy (AFM) (Dualscope/Rasterscope C26, DME, Herlev, Denmark) under a load of $0.2 \mathrm{nN}$ and a probe motion speed of $10 \mu \mathrm{m} / \mathrm{s}$.

\section{Silver distribution}

SEM-EDX (Scanning electron microscopy with an energy dispersive X-ray analytical system) analysis was performed to confirm the homogeneity of the distribution of the nano particles in the composite resins. Therefore, one disc-shaped sample from each test group was prepared for SEM-EDX analysis. Samples were broken into two pieces with a chisellike blade, and the broken surfaces were gold sputter coated (Sputter Coater, Emitech, K45OX Ashford, Kent, England) in a thin 15-nm layer to prevent the sample surfaces from burning during SEM observation. The elemental gold was finally eliminated from the diagram by the system software. The broken surfaces of each sample were observed with a scanning electron microscope.

\section{Wettability}

The wettability of the samples was assessed by measuring the contact angles of distilled water on composite resins with a contact angle measuring 
system (Sessile Drop, Kruss G10, Hamburg, Germany). The contact angle was defined as the angle at which the liquid interface met the solid surface of the composite disc at four points on each sample, and the mean of the points was reported as the contact angle of each sample. The surface of the drop was monitored constantly, and the contact angle was measured just after $20 \mathrm{~s}$, when the droplet was stabilized.

All data were analyzed by SPSS Software (Version 11, SPSS Inc., Chicago, USA) using two-way ANOVA and Tukey HSD multiple comparison tests. The level of significance was set at 0.05 .

\section{Results}

Figures 1 and 2 indicate the distribution of nano silver particles in composite resins. Figures 3 and 4 are the topographic images of the surface of composite resins taken by atomic force microscopy (AFM).

The means and standard deviations of the contact angles of the studied groups are summerized in Table 1.

Analysis of factorial variance was performed in

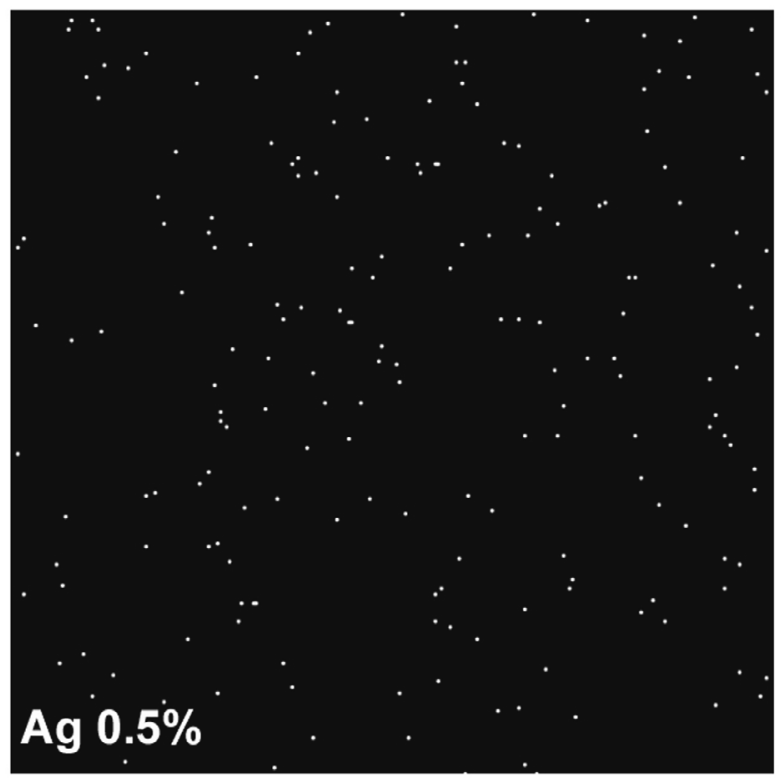

Magnification : $1054 \mathrm{X}$

Accelerating voltage ( $\mathrm{kV}$ ) : $\mathbf{2 0 . 0 0}$

Process time : 2

Sample is coated with Gold - thickness $(\mathrm{nm}): 15.0$, density $(\mathrm{g} / \mathrm{cm} 3): 19.32$

Figure 1 - Image of the surface of composites containing $0.5 \%$ nano silver particles. control and test groups for water contact angles. The contact angle was significantly different between the P90 and Z250 composites. The contact angle of the P90 composite was significantly higher than that of $\mathrm{Z} 250(\mathrm{p}=0.0001)$. The addition of nano silver particles reduced significantly the contact angle $(\mathrm{p}=0.0001)$. The interaction of the type of the composite and the concentration of the nano silver was significant $(\mathrm{p}=0.006)$. Therefore, exclusive of the type of composite, incorporation of different concentrations of nano silver reduced the contact angle significantly $(\mathrm{p}=0.008)$.

In the Z250 composite, increasing the concentration of nano silver particles from 0.5 to $1 \%$ caused

Table 1 - The contact angles of the composites (mean $\pm \mathrm{sd}$ ).

\begin{tabular}{c|c|c|c}
\hline \multirow{2}{*}{ Composite } & \multicolumn{3}{|c}{$\%$ of nano silver particle } \\
\cline { 2 - 4 } & $0 \%$ & $0.5 \%$ & $1 \%$ \\
\hline Z250 & $80.50 \pm 2.00 \mathrm{~A}, \mathrm{a}$ & $68.02 \pm 0.62 \mathrm{~A}, \mathrm{~b}$ & $74.37 \pm 0.22 \mathrm{~A}, \mathrm{c}$ \\
\hline P90 & $94.57 \pm 2.24 \mathrm{~B}, \mathrm{a}$ & $88.17 \pm 1.86 \mathrm{~B}, \mathrm{~b}$ & $88.32 \pm 2.91 \mathrm{~B}, \mathrm{c}$ \\
\hline
\end{tabular}

The different capital letters indicate statistically significant differences between the composites. Different lower case letters indicate statistically sig nificant differences between samples with and without nano silver.

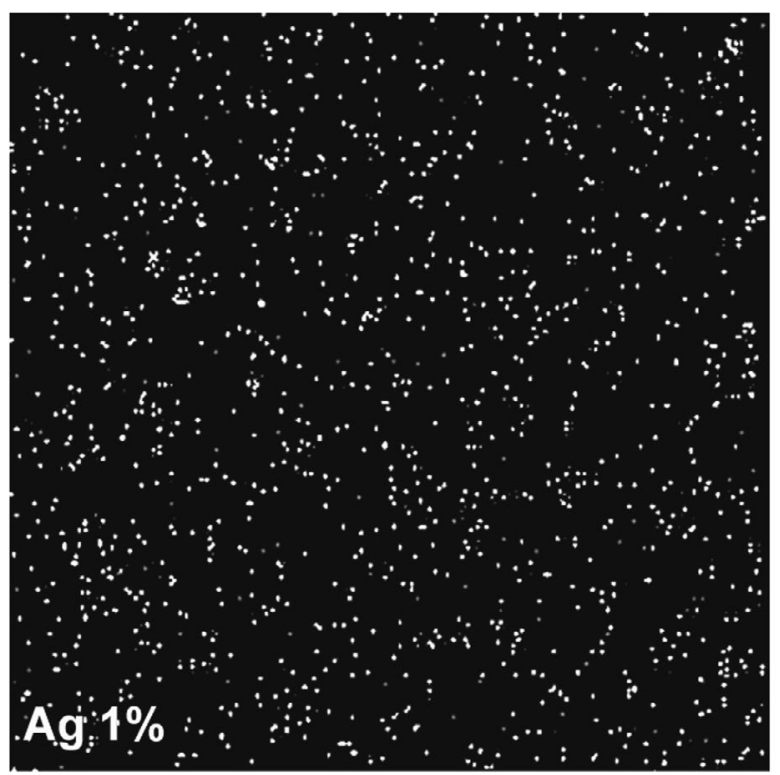

Magnification : $1054 \mathrm{X}$

Accelerating voltage ( $\mathrm{kV}$ ) : $\mathbf{2 0 . 0 0}$

Process time : 2

Sample is coated with Gold - thickness $(\mathrm{nm}): 15.0$, density $(\mathrm{g} / \mathrm{cm} 3): 19.32$

Figure 2 - Image of the surface of composites containing $1 \%$ nano silver particles. 
Figure 3 - Topographic image of the surface of the Z250 composite with atomic force microscopy (AFM).
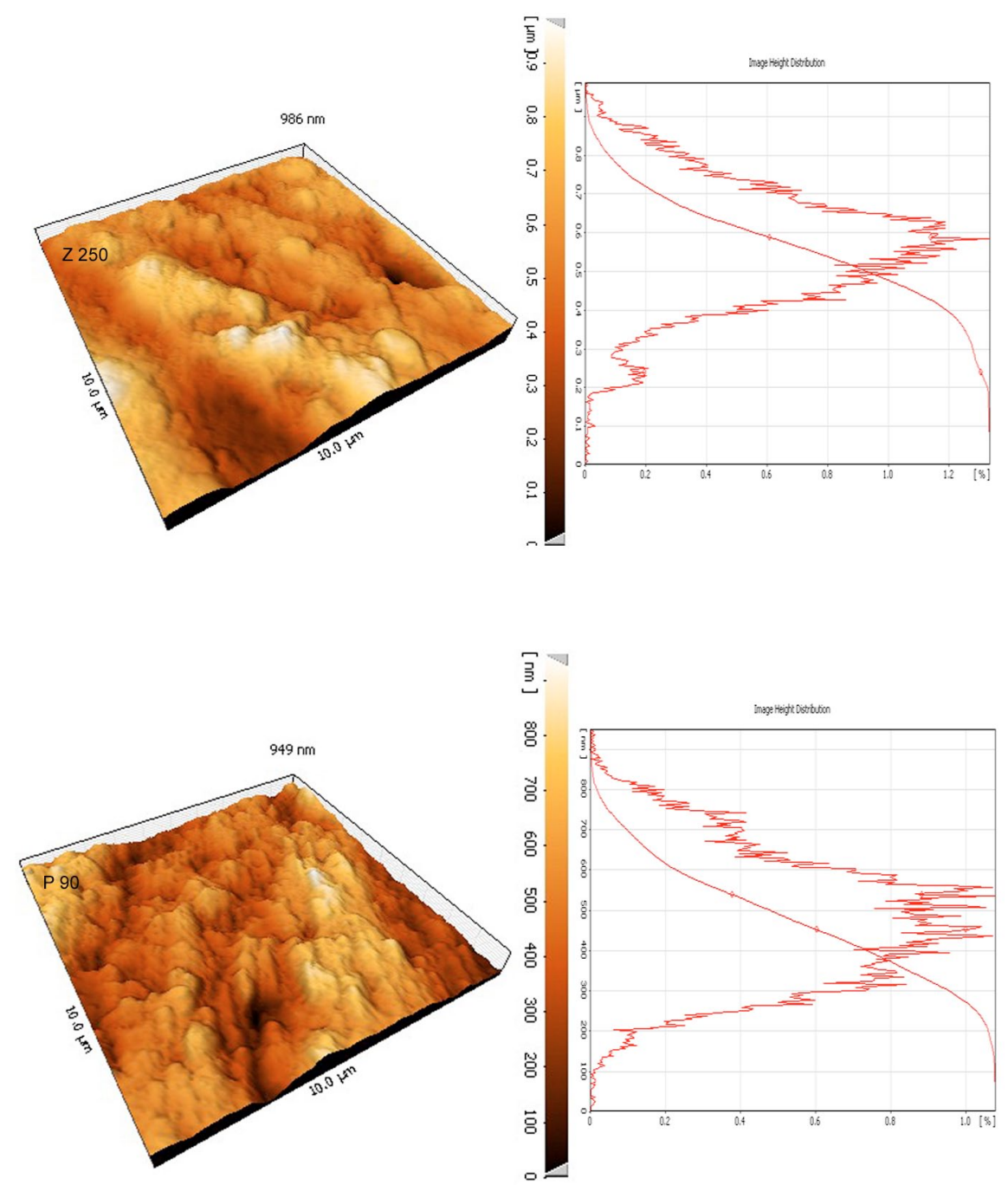

Figure 4 - Topographic image of the surface of the P90 composite with atomic force microscopy (AFM) an increase in the contact angle ( $\mathrm{p}=0.0001)$, whereas in the P90 composite the difference between the samples with $0.5 \%$ and $1 \%$ nano silver particles was not statistically significant $(\mathrm{p}>0.05)$.

\section{Discussion}

Considering the higher level of sorption of microorganisms by the composite resins compared to other restorative materials, ${ }^{12}$ some previous studies have suggested the addition of silver as an antibacterial material to the composite resin restorations. ${ }^{10,11}$ Silver is a safe bactericidal metal, because it is not toxic for animal cells, while it is severely toxic and lethal to bacterial cells. ${ }^{13,14}$

Concerns have been raised about the adverse effect of nano particle additives on the mechanical properties of composite resins. Previous studies have reported silver compounds added at $10 \%$ or greater to dental materials would significantly reduce compressive strength, elastic modulus, and tensile strength. ${ }^{11,15}$ Therefore, it seems that nano particles added in low concentrations, as in the present study, would not adversely affect the mechanical properties of composite resins. Discoloration and a change in color to a tone of gray are common problems in all materials containing silver, especially composite resins. ${ }^{16,17}$ Using low concentrations of metal nano particles can prevent severe discoloration of composite resins. ${ }^{16}$ It has been reported in a previous study that the incorporation of silver microparticulates at concentrations of $0.3 \%$ and $0.6 \%$ into the composite resin imparted antibacterial properties to the ma- 
terial. Therefore, according to previous studies, we chose concentrations of $0.5 \%$ and $1 \%$ silver nano particles to impart both the antibacterial properties of silver and the less detrimental effect of these nano particles on the color of composite resins.

In the present study, the influence of surface roughness was eliminated by polishing all specimens to a level of clinically acceptable surface smoothness $^{18}$ (about $1 \mu \mathrm{m}$ ), which was confirmed by AFM observations.

The contact angle of the silorane-based composite (P90) was significantly higher than that of the Z250 composite, which is indicative of the higher hydrophobicity of P90. This is in accordance with the study of Buergers et al. ${ }^{10}$ Addition of silver nano particles to P90 and Z250 composites caused a reduction in water contact angle of these composites. Both composites exhibited higher contact angles when the concentration of nano silver was increased from 0.5 to $1 \%$ by weight but the contact angles were still lower than that in the control groups. Therefore, depending on the type of the composite, addition of nano particles could significantly change (reduce) the water contact angle of the composites.

Contrary to the results of the present study, Bürgers et al. ${ }^{11}$ reported greater hydrophobicity upon the addition of silver to composite resins. They used a flowable composite in their study. Filler characteristics of composites have been considered a significant factor in their water sorption. The amount of water sorption by composite resin depends on the hydrophilicity of the polymeric matrices and the composition of the filler. There is also a correlation between filler load and water sorption. ${ }^{19}$ The flowable composite used in the study of Bürgers et al. (X-flow) contains $38 \%$ by volume silicon dioxide filler particles, whereas the Z250 composite is filled to $60 \%$ by volume with zirconia/silica particles, and Filtek Silorane contains $76 \%$ by volume quartz and yttrium fluoride filler particles. The contact angle of the flowable composite used in the study of Bürgers et al. ${ }^{11}$ was $66^{\circ}$, which increased upon the addition of silver particles. In addition, they used microparticulates of silver with sizes ranging from 3.5 to $18 \mu \mathrm{m}$. Metallic particles of silver have a large surface energy. The smaller the silver particles, the higher their energy and surface activity. Addition of nanometer sized silver particles, as done in the present study $(50 \mathrm{~nm})$ could result in an increase in the surface energy of composite resin, consequently decreasing the water contact angle. It is reported in previous studies that the addition of these particles to hydrophobic materials, such as composite resins, causes an increase in surface energy and a reduction of the contact angle, which is in accordance with the results of the present study. ${ }^{20}$

The results of our study indicated that the addition of nano silver could reduce the contact angle of the composite resins in comparison to the control groups; however, the water contact angle increased by increasing the nano silver concentration from 0.5 to $1 \%$ by weight. It is possible that the addition of more than $0.5 \%$ nano particles caused the accumulation of these particles, creating clusters of silver resembling microparticulate silver, and therefore resulting in an increase in hydrophobicity and contact angle in comparison with composites containing $0.5 \%$ nano silver.

Although the contact angle was reduced by the addition of nano silver to composite resins, numerous other factors affect bacterial adherence. The anti-adherence and bactericidal properties of nano silver-containing composites against mutans streptococci are reported in some previous studies. ${ }^{5,6,11,16,17}$

Although nano silver particles might be useful as an antibacterial agent incorporated into dental composites, this metal influences the color of the esthetic restorative materials and reduces water contact angles of the heavily filled composite resins. Therefore, addition of silver nano particles into composite resin restorations may not be clinically advantageous.

\section{Conclusions}

Within the limitations of the present study, it was concluded that addition of $0.5 \%$ and $1 \%$ silver nano particles increased the wettability of the methacrylate and silorane-based restorative composites.

\section{Acknowledgements}

The authors thank the Dental Research Center and Vice Chancellor of Research, Hamadan University of Medical Sciences, for supporting this study. 


\section{References}

1. Leprince J, Palin WM, Mullier T, Devaux J, Vreven J, Leloup G. Investigating filler morphology and mechanical properties of new low-shrinkage resin composite types. J Oral Rehabil. 2010 May;37(5):364-76.

2. Klautau EB, Carneiro KK, Lobato MF, Machado SM, Silva e Souza Jr MH. Low shrinkage composite resins: influence on sealing ability in unfavorable C-factor cavities. Braz Oral Res. 2011 Jan-Feb;25(1):5-12.

3. Ilie N, Jelen E, Clementino-Luedemann T, Hickel R. Lowshrinkage composite for dental application. Dent Mater J. 2007 Mar;26(2):149-55.

4. Weinmann W, Thalacker C, Guggenberger R. Siloranes in dental composites. Dent Mater. 2005 Jan;21(5):68-74.

5. Kondo Y, Takagaki T, Okuda M, Ikeda M, Kadoma Y, Yamauchi J, et al. Effect of PMMA filler particles addition on the physical properties of resin composite. Dent Mater J. 2010 Oct;29(5):596-601.

6. Namen FM, Galan Jr J, Oliveira JF, Cabreira RD, Costa E, Silva-Filho F, et al. Surface properties of dental polymers: measurements of contact angles, roughness and fluoride release. Mater Res. 2008 Jul-Sep;11(3):239-43.

7. Beyth N, Domb AJ, Weiss EI. An in vitro quantitative antibacterial analysis of amalgam and composite resins. J Dent. 2007 Mar;35(3):201-6.

8. Sarrett DC. Prediction of clinical outcomes of a restoration based on in vivo marginal quality evaluation. J Adhes Dent. 2007;9(Suppl. 1):117-20.

9. Spacciapoli P, Buxton D, Rothstein D, Friden P. Antimicrobial activity of silver nitrate against periodontal pathogens. J Periodontol Res. 2001;36(2):108-13.

10. Buergers R, Schneider-Brachert W, Hahnel S, Rosentritt M, Handel G. Streptococcal adhesion to novel low-shrink silorane-based restorative. Dent Mater. 2009 Feb;25(2):269-75.
11. Bürgers R, Eidt A, Frankenberger R, Rosentritt M, Schweikl $\mathrm{H}$, Handel G, et al. The anti-adherence activity and bactericidal effect of microparticulate silver additives in composite resin materials. Arch Oral Biol. 2009 Jun;54(6):595-601.

12. Papagiannoulis L, Kakaboura A, Eliades G. In vivo vs in vitro anticariogenic behavior of glass-ionomer and resin composite restorative materials. Dent Mater. 2002 Dec;18(8):561-9.

13. Janardhanan R, Karuppaiah M, Hebalkar N, Narsinga Rao T. Synthesis and surface chemistry of nano silver particles. Polyhedron. 2009 Aug;28(12):2522-30.

14. Fondevila M, Herrer R, Casallas MC, Abecia L, Ducha JJ. Silver nanoparticles as a potential antimicrobial additive for weaned pigs. Anim Feed Sci Technol. 2009 Apr;150(3):259-69.

15. Yoshida K, Tanagawa M, Atsuta M. Characterization and inhibitory effect of antibacterial dental resin composites incorporating silver-supported materials. J Biomed Mater Res. 1999 Dec;15(4):516-22.

16. Hernández-Sierra JF, Ruiz F, Pena DC, Martínez-Gutiérrez F, Martínez AE, Guillén Ade J, et al. The antimicrobial sensitivity of Streptococcus mutans to nanoparticles of silver, zinc oxide, and gold. Nanomedicine. 2008 Sep;4(3):237-40.

17. Li L, Deng J, Deng H, Liu Z, Li X. Preparation, characterization and antimicrobial activities of chitosan $/ \mathrm{Ag} / \mathrm{ZnO}$ blend films. Chem Eng J. 2010 May;160(1):378-82.

18. Sidhu SK, Henderson LJ. The surface finish of light-cured composite resin materials. Clin Mater. 1993;12(1):11-5.

19. Berger SB, Palialol AR, Cavalli V, Giannini M. Characterization of water sorption, solubility and filler particles of lightcured composite resins. Braz Dent J. 2009;20(4):314-8.

20. Torchinsky I, Rosenman G. Wettability modification of nanomaterials by low-energy electron flux. Nanoscale Res Lett. 2009 Jul;4(10):1209-17. 